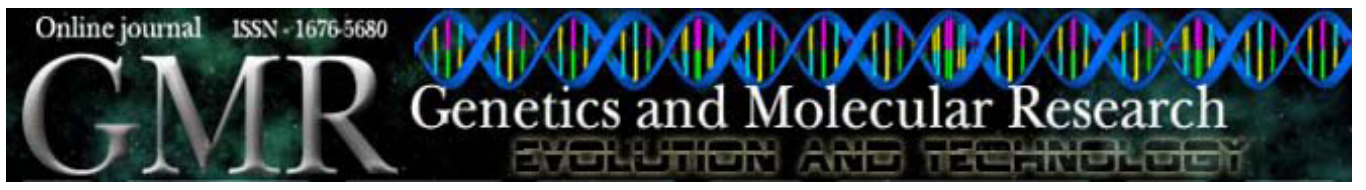

\title{
Heteropicnotic chromatin and nucleolar activity in meiosis and spermiogenesis of Limnogonus aduncus (Heteroptera, Gerridae): a stained nucleolar organizing region that can serve as a model for studying chromosome behavior
}

\author{
M.M.U. Castanhole ${ }^{1}$, L.L.V. Pereira ${ }^{1}$, H.V. Souza ${ }^{1}$, \\ H.E.M.C. Bicudo ${ }^{1}$, L.A.A. Costa ${ }^{2}$ and M.M. Itoyama ${ }^{1}$
}

${ }^{1}$ Departamento de Biologia, Laboratório de Citogenética de Insetos, Instituto de Biociências, Letras e Ciências Exatas,

Universidade Estadual Paulista, São José do Rio Preto, SP, Brasil

${ }^{2}$ Museu Nacional, Departamento de Entomologia, Rio de Janeiro, RJ, Brasil

Corresponding author: M.M. Itoyama

E-mail: mary@ibilce.unesp.br

Genet. Mol. Res. 7 (4): 1398-1407 (2008)

Received October 6, 2008

Accepted October 15, 2008

Published December 16, 2008

\begin{abstract}
Males of Limnogonus aduncus were found to have the sex chromosome system X0 and chromosome number $2 \mathrm{n}=23$ $(22 \mathrm{~A}+\mathrm{X} 0)$. Testis cells were stained with lacto-acetic orcein and silver nitrate so that changes in the morphology and degree of staining of the heteropicnotic chromatin and the nucleolar material could be observed during meiosis and spermiogenesis. These structures share the same nuclear position and could be seen until almost the end of spermiogenesis. A chromosome region stained with silver ni-
\end{abstract}


trate was indicative of a nucleolar organizing region (NOR), which is rarely detected in Heteroptera with this technique. The NOR is located at one end of a single member of an autosome pair. The finding of this stained region enabled us to observe that the telomeric association of sister chromatids that characterizes the Heteroptera does not include the chromosome ends, where NORs are located; we also observed in anaphase that the chromosome end through which it is pulled to the pole is the one containing the NOR. Another observation was that the single nucleolar body present in the cells at anaphase never goes to the cell pole that does not receive the NOR. We conclude that L. aduncus is a good model for cytogenetic studies involving nucleolar activity and also may be useful for studying the mechanisms of activation and inactivation of kinetic activity at the chromosome ends. Although the chromosomes of Heteroptera are known to be holocentric, whether kinetic activity is restricted to one or involves both chromosome ends is still not well understood.

Key words: Stained nucleolar organizing region; Aquatic insects; Telomeric association; Kinetic activity of the chromosome ends

\section{INTRODUCTION}

The Heteroptera is a numerically large and highly diverse group of insects. About 40,000 species from this suborder have been described, many of which are pests of food and are often economically important. These insects are highly variable cytogenetically. Chromosome numbers vary from 4 to 80 , and the mechanisms of sex determination are simple $(\mathrm{XY} / \mathrm{XX}$ or $\mathrm{X} 0 / \mathrm{XX})$, multiple $(\mathrm{Xn} 0 / \mathrm{XnXn}, \mathrm{XnY} / \mathrm{XnXn}$ and $\mathrm{XYn} / \mathrm{XX}$ ) or a neoXY system (Chickering and Bacorn, 1933; Schrader, 1940; Jande, 1959; Ueshima, 1979; Manna, 1984). The chromosomes are holocentric. In meiosis, which is achiasmatic or chiasmatic, the autosomes go through a pre-reductional division, while in the sex chromosomes division is post-reductional. The karyotype may include microchromosomes, B chromosomes or univalent chromosomes (Ueshima, 1979; Manna, 1984; Papeschi and Mola, 1990; González-Garcia et al., 1996; Grozeva and Nokkala, 1996; Suja et al., 2000; Nokkala and Nokkala, 2004). Nucleolar organizer regions (NORs) have been detected in a small number of species; in all cases their location was subterminal or median, occurring in a single pair of autosomes or in the sex chromosomes (Camacho et al., 1985; Fossey and Liebenberg, 1995; González-García et al., 1996; Papeschi and Bressa, 2002; Papeschi et al., 2003; Rebagliati et al., 2003).

Limnogonus aduncus, a member of the Gerridae family of Heteroptera, are surface-skimming aquatic insects. Cytogenetic information on the Gerridae is scarce. However, it is known that they are characterized by modal diploid chromosome numbers of 21 $(20 \mathrm{~A}+\mathrm{X} 0)$ or $23(22 \mathrm{~A}+\mathrm{X} 0)$, sometimes including microchromosomes (Ueshima, 1979). The X0 sex chromosome system predominates among species in this family, but there are some species with an XY system. In a polar view, the metaphase chromosomes seem to be arranged in a ring shape, at the equatorial region. Information regarding morphological 
variation of heteropicnotic chromatin and nucleolar pattern during spermatogenesis was until now unavailable for the Gerridae family.

\section{MATERIAL AND METHODS}

Twenty specimens classified as L. aduncus were collected from the Municipal reservoir of São José do Rio Preto, São Paulo State, Brazil, using a net. The specimens were transported in flasks with water to the laboratory and separated by sex, and the 13 males were fixed in methanol:acetic acid $(3: 1)$ and kept at $4^{\circ} \mathrm{C}$. The preparations were done by crushing the testes of each insect on a slide, followed by staining with the usual lacto-acetic orcein technique or with silver nitrate impregnation (Howell and Black, 1980), which preferentially stains the nucleolar material. Sequential staining using both techniques was tried without success, no matter which technique was used first. The images were captured on a Zeiss microscope using the AXIO VISION program for image analysis.

\section{RESULTS AND DISCUSSION}

Some Heteroptera are characterized by multilobed testes wrapped in a reddish membrane. This characteristic is common in the Pentatomidae family, in which the standard number is seven lobes. Variations may occur, such as in Antitheucus tripterus, which has six lobes (Souza et al., 2007a). We found that L. aduncus (Gerridae) also has multilobed testes. Two elongated lobes, wrapped in a transparent membrane, were found. The male sex chromosome system of L. aduncus is X0 and its chromosome complement is $2 \mathrm{n}$ $=23(22 \mathrm{~A}+\mathrm{X} 0)$. This number of chromosomes and the sex chromosome system were also detected in another species of the same genus, L. fossarum (Banerjee, 1958).

\section{Preparations stained with lacto-acetic orcein}

Polyploid nutritive cells of L. aduncus testes had more than 100 heteropicnotic chromatin bodies heavily stained by orcein (Figure 1a). In spermatogonial cells at the beginning of prophase I (leptotene), a single strongly stained region was observed, probably formed by the sex chromosomes, which remain condensed until pachytene (Figure 1b-d). Terminal or interstitial chiasmata were detected in diplotene and diakinesis (Figures 1e-i, $2 a)$. One of the chromatids of the largest autosome pair showed telomeric association with a chromatid of another pair; this could be seen until the end of prophase I (Figure 1e-i). Another autosome pair showed a constriction that remained visible until diplotene (Figure $1 \mathrm{e}, \mathrm{f})$. After diplotene, the cells enter the diffuse stage, greatly increasing the nucleus size and decreasing chromatin condensation (Figure $2 \mathrm{~b}$ ).

As known from other Heteroptera, in a polar view, cells of $L$. aduncus males at metaphase I show the autosomes arranged in a ring shape, with the sex chromosomes located in the internal part, while in a lateral view they appear vertically aligned in the equatorial region of the cell (Figure 2c). The metaphase chromosomes of $L$. aduncus form interconnected chromosome groups joined by chromatin threads (Figure 2d). Telophase cells are large and show abundant chromatin with a network aspect. There are two major heteropicnotic formations interspersed in this background, one at each cell pole (Figure 2e). 

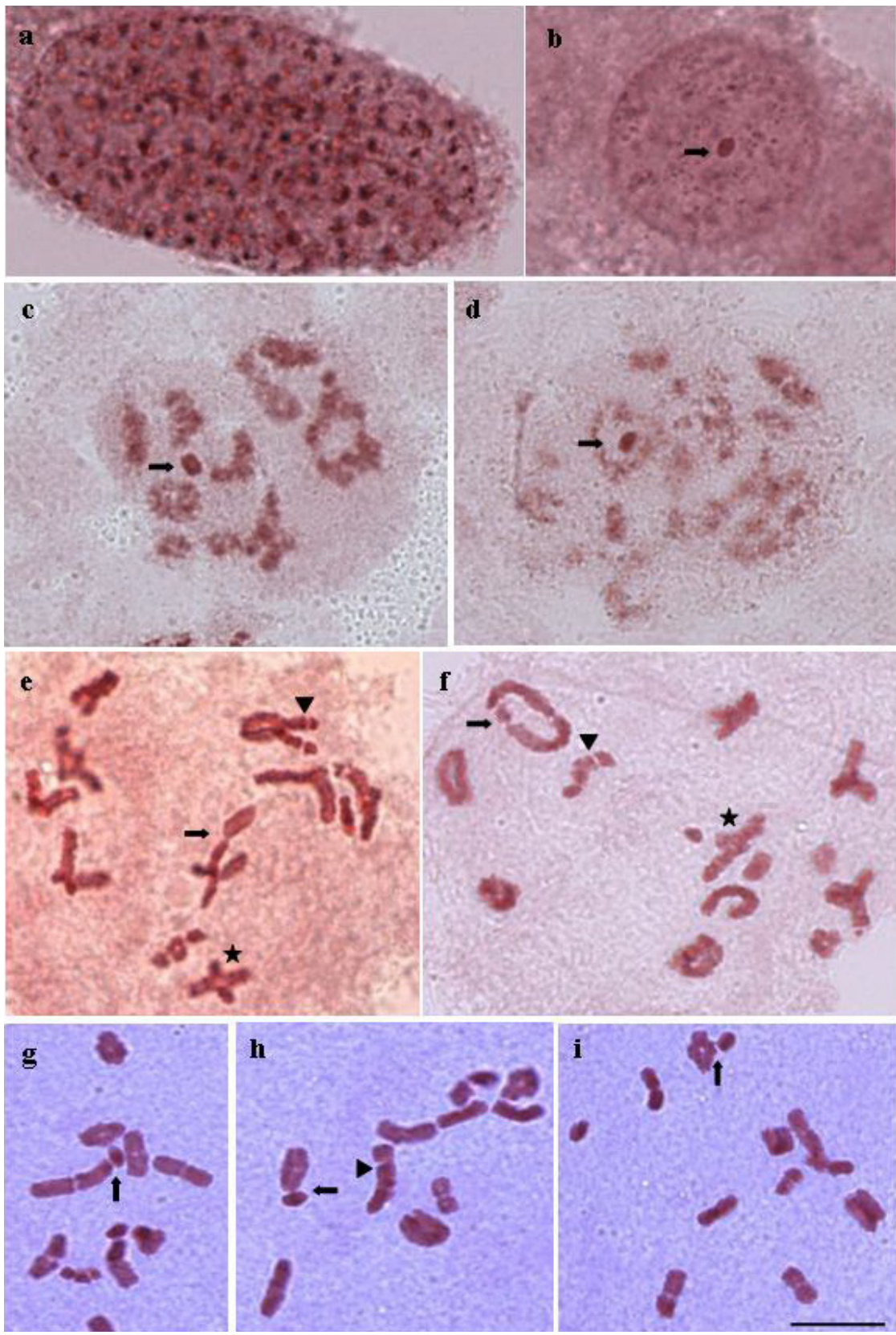

Figure 1. Cells of the seminiferous tubules of Limnogonus aduncus stained with lacto-acetic orcein. (a) Nutritive polyploid cell showing many heteropicnotic regions, (b) prophase I (leptotene) with a relocated heteropicnotic region (arrow), (c,d) cells in pachytene showing a halo around the single heteropicnotic region (arrows), (e-i) diplotene: a constriction is observed in an autosome pair (arrowhead e, $\mathrm{f}, \mathrm{h}$ ), two autosomes are in telomeric association (arrow, e-i) and an autosome shows an interstitial chiasma (asterisk, e, f). Bar: $10 \mu \mathrm{m}$. 

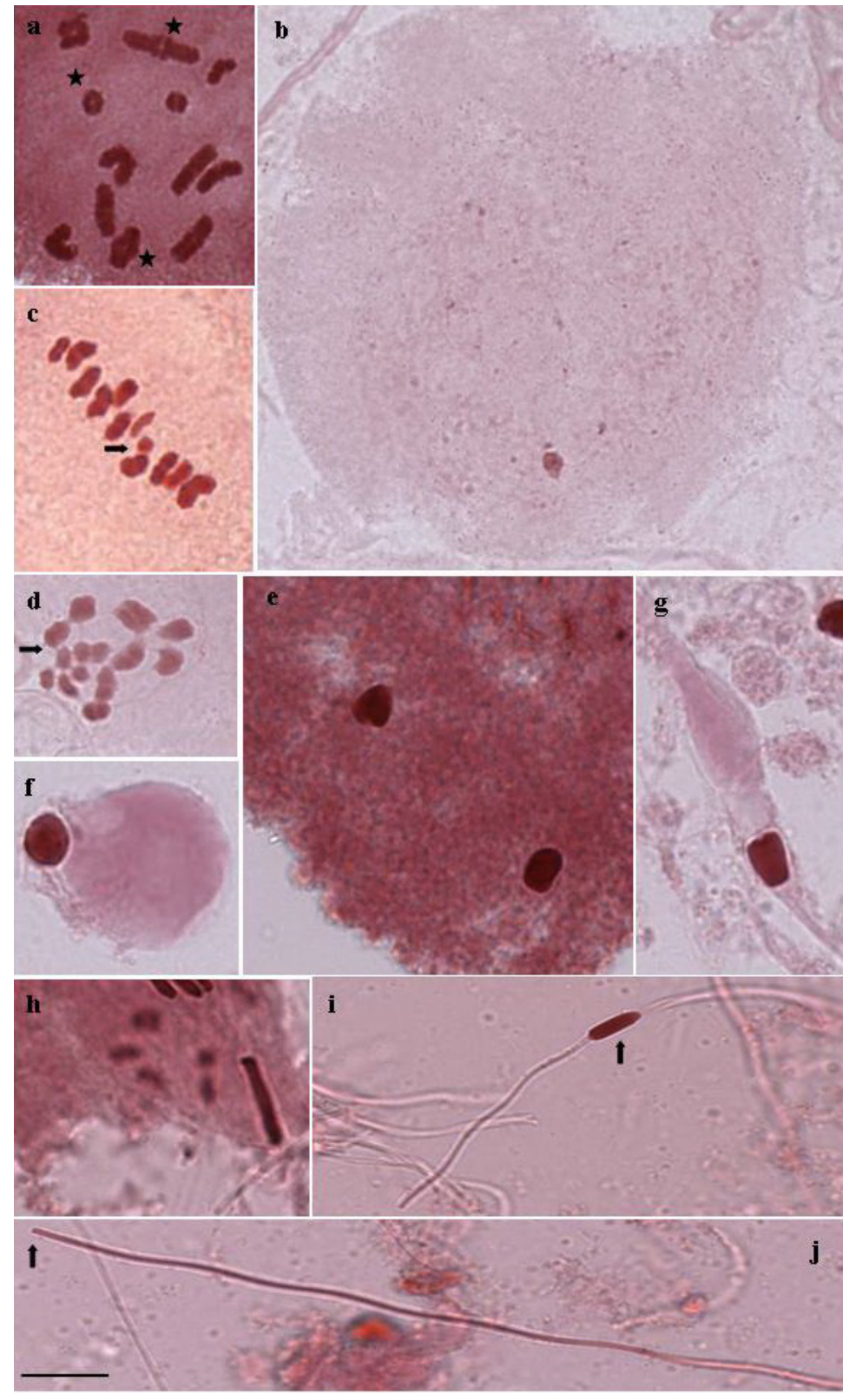

Figure 2. Cells of the seminiferous tubules of Limnogonus aduncus stained with lacto-acetic orcein. (a) Cell in diakinesis showing terminal or interstitial chiasmas (asterisks), (b) cell in diffuse stage, (c) metaphase I showing the 22 autosomes and the $\mathrm{X}$ chromosome (arrow), (d) metaphase I: chromosomes joined by chromatin threads (arrow), (e) cell in telophase with two major heteropicnotic regions, (f) rounded spermatid showing a large heteropicnotic region located at the nucleus edge, $(\mathrm{g})$ spermatid in elongation: note the change in morphology of the heteropicnotic material, (h) rod-shaped spermatid with strong staining, (i) spermatid head with heteropicnotic material located in the posterior region (arrow), (j) spermatid in elongation (arrow indicating the region of the head). Bar: $10 \mu \mathrm{m}$. 
At the end of the meiotic process, the round spermatids carry a single heteropicnotic formation, located at the nucleus edge (Figure 2f). As the spermatid nucleus elongates, this heteropicnotic material also assumes an elongated shape, which is seen in the posterior region of the spermatid head (Figure 2g). In a later stage, the spermatid heteropicnotic region is rod shaped (Figure 2h). During development, this region stands out within the head of the spermatids, due to its diameter (Figure 2i). Almost at the end of the spermiogenesis process, the spermatids have small head and a long tail, and the heteropicnotic region is no longer visible (Figure 2j).

\section{Preparations impregnated by silver ions}

Nuclei of the nutritive polyploid cells of the testes had many regions impregnated by silver ions, indicating that they are made of nucleolar material (Figure 3a). Some of them were markedly larger, but this may be due to the joining of smaller formations. The spermatogonial cells at the beginning of prophase show two nucleoli of very different sizes, one of them being about eight times the size of the other (Figure 3b).

In some organisms, silver impregnation stains not only the nucleolus, but also NORs, located at specific points of certain chromosomes. The stained NORs are those that were active during the previous interphase. The reason why these regions are silver stained in some organisms and not in others is still unknown. Silver-stained NORs have been rarely observed in Heteroptera species, but in L. aduncus, between diplotene and diakinesis, we start to see a terminal NOR in both sister chromatids of a single homologous chromosome of an autosome pair (Figure 3c-g). This pattern of NOR staining has been observed in some species of the Heteroptera families, Coreidae (Fossey and Liebenberg, 1995; Papeschi et al., 2003; Cattani et al., 2004), Pentatomidae (Camacho et al., 1985; Gonzalez-Garcia et al., 1996; Rebagliati et al., 2003) and Belostomatidae (Papeschi and Bressa, 2002).

At the beginning of metaphase I, the NOR is more strongly stained (Figures 3g,h, 4a). Data on other organisms, available in the literature (Bardella et al., 2008), support the hypothesis that in L. aduncus the NOR of a single chromosome member of the pair is active in the interphase. Also on the basis of data in the literature (Lanzone and Souza, 2006), we believe that the chromosome pair, which shows the constriction, is the same pair that in Heteropteran genus Antiteuchus carries the NOR.

Several studies have provided evidence that during meiotic division of Heteroptera the kinetic activity is restricted to the ends of the chromosomes (Schrader, 1935; Hughes-Schrader and Schrader, 1961). Camacho et al. (1985) observed a bivalent monochiasmatic chromosome of Nezara viridula (Pentatomidae); they concluded that only the chromosome end farthest from the chiasma has kinetic activity. On the other hand, Pérez et al. (1997) observed in Triatoma infestans that both ends of the bivalent 3 have kinetic activity and that, in different metaphases, the kinetically active end varies; they also mentioned that even the end closest to chiasmas shows kinetic activity. A problem that arises from these observations on the kinetic activity of the chromosome ends is that the mechanism that determines whether they are active is unknown (Pérez et al., 1997).

The stained NOR of $L$. aduncus enabled us to observe that although the two chromatids of the same chromosome show telomeric association with each other in metaphase and anaphase I (telomeric association is also a feature of the other Heteroptera), this association does not occur in the chromatid end carrying NOR. The end carrying NOR was only observed directed towards one of the cell poles, seeming to be the end at which the sister chromatids 

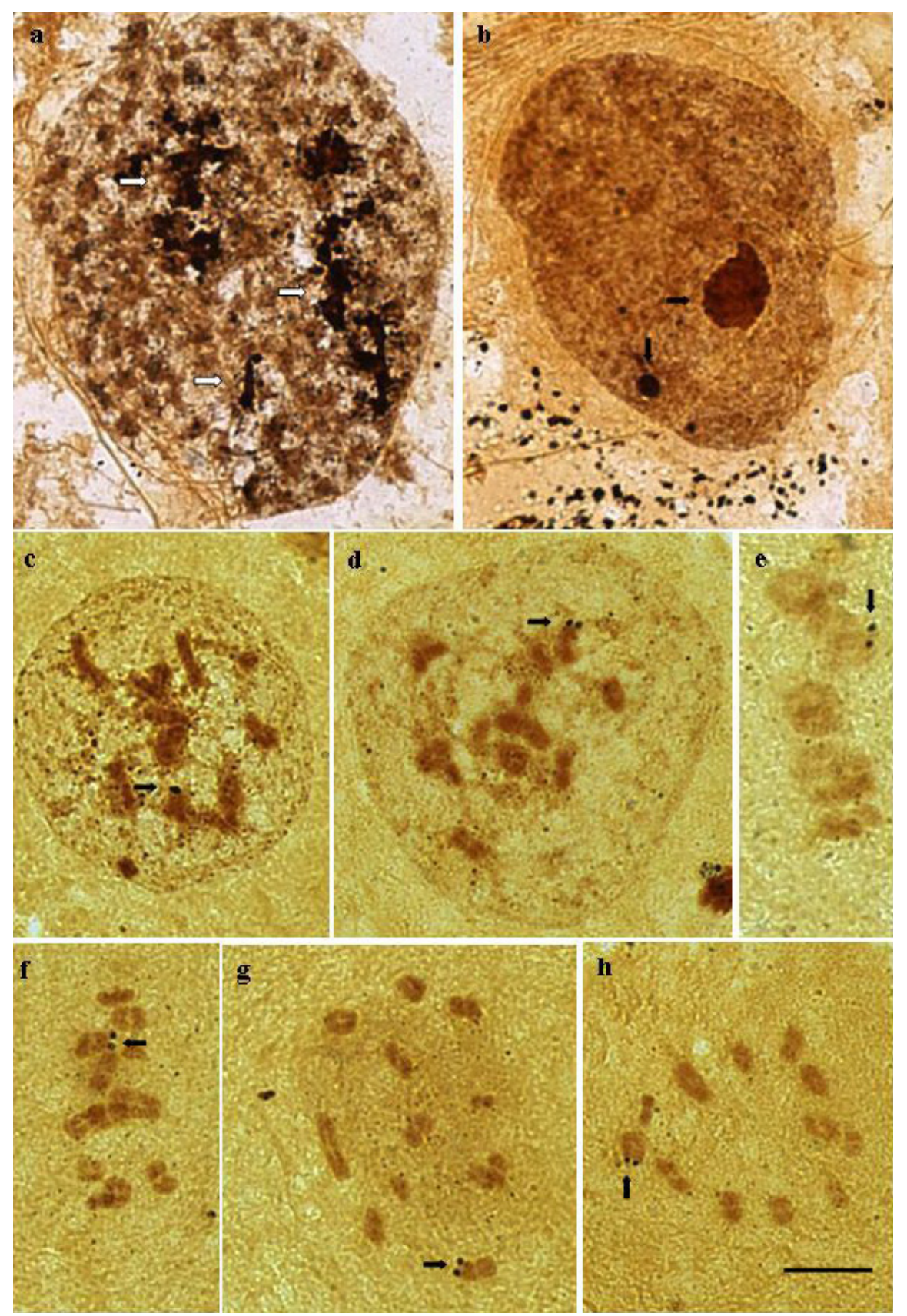

Figure 3. Cells of the seminiferous tubules of an adult Limnogonus aduncus impregnated with silver nitrate. (a) Nutritive polyploid cell showing many impregnated regions (arrows), (b) initial prophase (leptotene) with two nucleolar bodies of different sizes (arrows), (c-g) cells in different stages of prophase I showing the nucleolar organizing region stained in an autosome (arrows), (h) cells in the final stage of prophase also showing the stained NOR (arrow). Bar: $10 \mu \mathrm{m}$. 

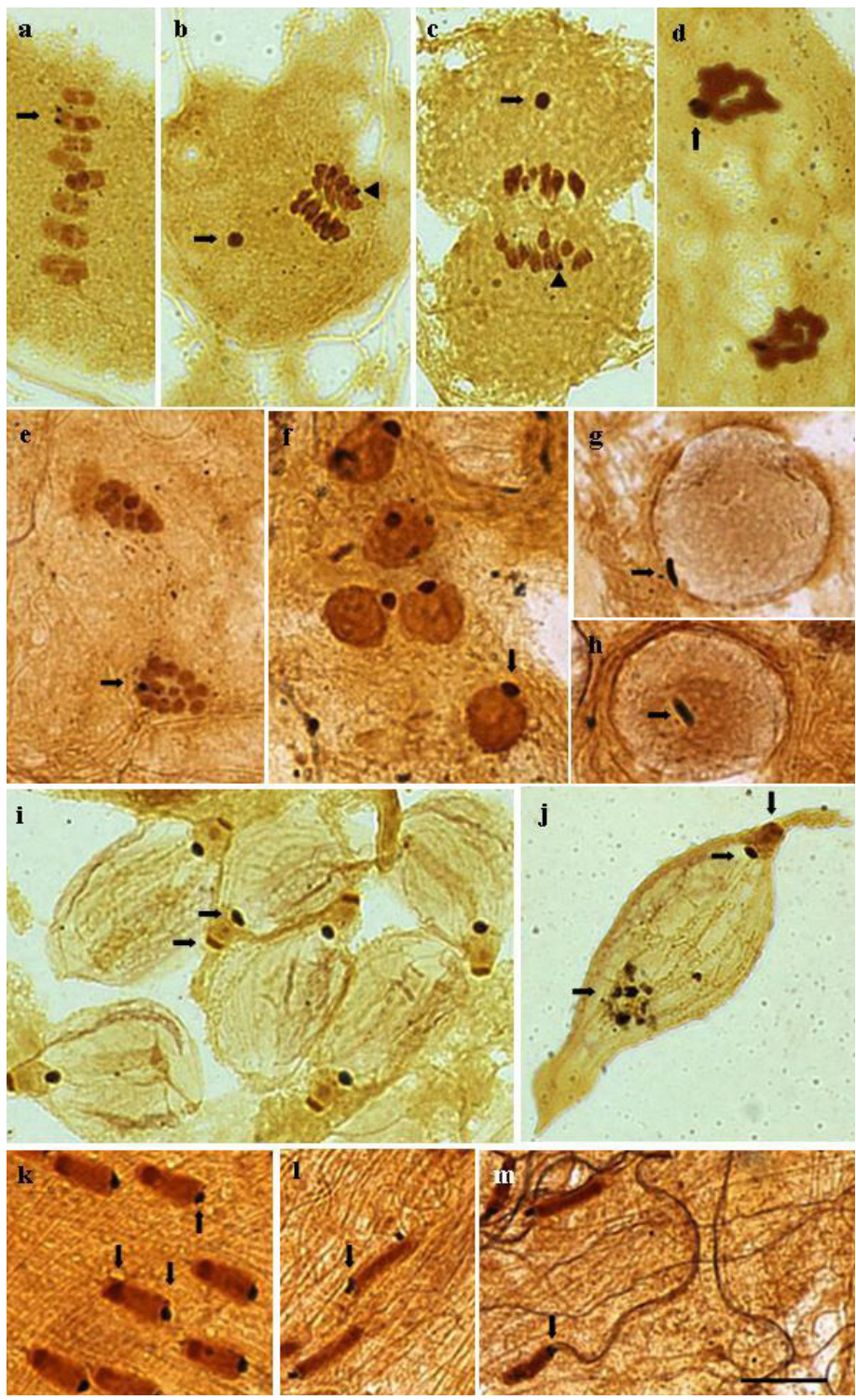

Figure 4. Cells of the seminiferous tubules of an adult Limnogonus aduncus impregnated with silver nitrate. (a) Metaphase I in side view showing an autosome with a stained NOR (arrow) and the telomeric association of chromosomes. $(\mathrm{b}, \mathrm{c})$ Anaphase I with a nucleolus (arrow) present in the daughter cell that did not receive the chromosome with the stained NOR (arrowhead), $(\mathrm{d}, \mathrm{e})$ telophase: a nucleolus is present only in one daughter cell (arrow), (f) rounded spermatid with a nucleolus located at the nucleus edge (arrow), (g, h) spermatid with elongate nucleolus (arrows), (i) spermatid with three silver stained formations inserted in a less strongly silver stained region, located in the posterior region of the head (arrows), (j) silver impregnated bodies in the anterior region of the spermatid head (arrow) and maintenance of the nucleolar structure located in the posterior region of the head (arrows); (k-m) spermatid in elongation, at the beginning showing staining in both the posterior and anterior regions of the head; as the development proceeds, the staining becomes restricted to the posterior region (arrows). Bar: $10 \mu \mathrm{m}$. 
are linked to the spindle fibers to be pulled to the poles. Thus, at least for this NOR-bearing chromosome, apparently only one chromosome end presents kinetic activity. Whether there is any association of the kinetic activity with NOR was not resolved in our study.

At the onset of anaphase I, we found only one nucleolar body in the cell (Figure 4b,c), which we also saw at telophase (Figure 4d,e). After migration of chromosomes to the cell poles, the nucleolus was always present at the pole that did not receive the chromosome pair carrying NOR (Figure 4c). So, apparently, the cell that does not receive the nucleolus is the one able to synthesize it through the active NOR. These observations suggest an imprinting process in NOR activity, a possibility that deserves further study.

At the beginning of spermiogenesis, the round spermatids show the single nucleolus located in the periphery of the nucleus (Figure 4f). As the process proceeds, the nucleolus elongates and locates at one of the nucleus poles (Figure 4g,h). As spermatid lengthening continues, there is a change in the nucleolus morphology, as if the nucleolar structure had been divided into three formations that appear included in a weakly silver-impregnated region. Two of the three formations are located at the ends of this region, and the third is in the intermediate space. The intermediate formation is less intensely stained than the other two. This entire nucleolar structure is located in the posterior region of the sperm head (Figure 4i). As the differentiation proceeds, several silver impregnated bodies are also observed in the anterior region of the sperm head (Figure $4 \mathrm{j}$ ). In the final stages of spermiogenesis, the anterior region of the sperm head shows less stained nucleolar material, while a more strongly stained body is seen in its posterior region (Figure 4k-m). Silver-stained nucleolar material occurring in the sperm head has also been described in the Heteropteran families, Lygaeidae and Pentatomidae (Souza et al., 2007a,b).

Although we were not successful in the attempt to use both lacto-acetic orcein and silver impregnation sequentially, it was possible to observe by image comparison that the locations of the heterochromatin and the nucleolar material coincide. This is quite visible in the figures of the spermatids: Figures $2 \mathrm{f}$ (orcein) and $4 \mathrm{f}$ (silver nitrate) and Figures $2 \mathrm{~g}$ (orcein) and $4 \mathrm{j}$ (silver nitrate). A halo is seen around heteropicnotic bodies in Figure 1c, d; a similar structure observed in Triatoma species was shown to be nucleolar material (Severi-Aguiar et al., 2006). The close approximation of heterochromatin and NORs is well known in the literature and reflects functional interaction of these structures. This proximity involves, among other phenomena, the mediation of the activity of rDNA genes (Santoro et al., 2002). In Triatoma vitticeps, the coincident locations of these structures were also described in testes (Severi-Aguiar et al., 2006). We conclude that L. aduncus could be used as a model for cytogenetic studies.

\section{ACKNOWLEDGMENTS}

Special thanks to Dr. Sonia Maria Oliani of the Department of Biology, IBILCE/ UNESP for allowing us to use the image system of her laboratory, and to FAPESP (Fundação de Amparo à Pesquisa do Estado de São Paulo), FUNDUNESP (Fundação para o Desenvolvimento da UNESP) and CAPES (Coordenação de Aperfeiçoamento de Pessoal de Nível Superior) for financial support.

\section{REFERENCES}

Banerjee MK (1958). A study of the chromosomes during meiosis in twenty-eight species of Hemiptera (Heteroptera, 
Homoptera). Proc. Zool. Soc. 11: 9-31.

Bardella VB, Azeredo-Oliveira MT and Tartarotti E (2008). Cytogenetic analysis in the spermatogenesis of Triatoma melanosoma (Reduviidae; Heteroptera). Genet. Mol. Res. 7: 326-335.

Camacho JPM, Belda J and Cabrero J (1985). Meiotic behaviour of the holocentric chromosomes of Nezara viridula (Insecta, Heteroptera) analysed by C-banding and silver impregnation. Can. J. Genet. Cytol. 27: 490-497.

Cattani MV, Greizerstein EJ and Papeschi AG (2004). Male meiotic behavior and nucleolus organizer regions in Camptischium clavipes (Fabr.) (Coreidae, Heteroptera) analyzed by fluorescent banding and in situ hybridization. Caryologia 57: 267-273.

Chickering AM and Bacorn B (1933). Spermatogenesis in the Belatomatidae. IV. Multiple chromosomes in Lethocerus. Pap. Mich. Acad. Sci. Arts Lett. 17: 529-534.

Fossey A and Liebenberg H (1995). Meiosis and nucleolar structures in the stink bug Carlisis wahlbergi Stal (Coreidae: Heteroptera). Cytobios 81: 7-15.

González-Garcia JM, Antonio C, Suja JA and Rufas JS (1996). Meiosis in holocentric chromosomes: kinetic activity is randomly restricted to the chromatid ends of sex univalents in Graphosoma italicum (Heteroptera). Chromosome Res. 4: 124-132.

Grozeva S and Nokkala S (1996). Chromosomes and their meiotic behavior in two families of the primitive infraorder Dipsocoromorpha (Heteroptera). Hereditas 125: 31-36.

Howell WM and Black DA (1980). Controlled silver-staining of nucleolus organizer regions with a protective colloidal developer: a 1-step method. Experientia 36: 1014-1015.

Hughes-Schrader S and Schrader F (1961). The kinetochore of the Hemiptera. Chromosoma 12: 327-350.

Jande SS (1959). An analysis of the chromosomes in four species of the family Belostomatidae (Heteroptera, Cryptocerata). Res. Bull. Panjab Univ. Sci. 10: 25-34.

Lanzone C and Souza MJD (2006). C-banding, fluorescent staining and NOR location in holokinetic chromosomes of bugs of the Neotropical genus Antiteuchus (Heteroptera: Pentatomidae: Discocephalinae). Eur. J. Entomol. 103: 239-243.

Manna GK (1984). Chromosomes in Evolution in Heteroptera. In: Chromosomes in Evolution of Eukaryotic Groups (Sharma AK, ed.). CRC Press, Boca Raton, 189-225.

Nokkala S and Nokkala C (2004). Interaction of B chromosomes with A or B chromosomes in segregation in insects. Cytogenet. Genome Res. 106: 394-397.

Papeschi AG and Mola LM (1990). Meiotic studies in Acanonicus hahni (Stal) (Coreidae, Heteroptera). I. Behaviour of univalents in desynaptic individuals. Genetica 80: 31-38.

Papeschi AG and Bressa MJ (2002). Cytogenetic Studies in Belostomatidae from Argentina. Abstracts of the Second Quadrennial Meeting of the International Heteropterists Society, St. Petersburg, 46.

Papeschi AG, Mola LM, Bressa MJ, Greizerstein EJ, et al. (2003). Behaviour of ring bivalents in holokinetic systems: alternative sites of spindle attachment in Pachylis argentinus and Nezara viridula (Heteroptera). Chromosome Res. 11: 725-733.

Pérez R, Panzera F, Page J, Suja JA, et al. (1997). Meiotic behaviour of holocentric chromosomes: orientation and segregation of autosomes in Triatoma infestans (Heteroptera). Chromosome Res. 5: 47-56.

Rebagliati P, Papeschi AG and Mola LM (2003). Meiosis and fluorescent banding in Edessa meditabunda and E. rufomarginata (Heteroptera: Pentatomidae: Edessinae). Eur. J. Entomol. 100: 11-18.

Santoro R, Li J and Grummt I (2002). The nucleolar remodeling complex NoRC mediates heterochromatin formation and silencing of ribosomal gene transcription. Nat. Genet. 32: 393-396.

Schrader F (1935). Notes on the mitotic behavior of long chromosomes. Cytology 6: 422-430.

Schrader F (1940). The formation of tetrads and the meiotic mitoses in the male of Rhytidolomia senilis Say (Hemiptera, Heteroptera). J. Morphol. 67: 123-141.

Severi-Aguiar GD, Lourenco LB, Bicudo HE and Azeredo-Oliveira MT (2006). Meiosis aspects and nucleolar activity in Triatoma vitticeps (Triatominae, Heteroptera). Genetica 126: 141-151.

Souza HV, Bicudo HEMC, Costa LAA and Itoyama MM (2007a). A study of meiosis and spermiogenesis in the testicular lobes of Antiteuchus tripterus (Heteroptera: Pentatomidae). Eur. J. Entomol. 104: 353-362.

Souza HV, Bicudo HE and Itoyama MM (2007b). Study of chromosomal and nucleolar aspects in testes of Nysius californicus (Heteroptera: Lygaeidae). Genet. Mol. Res. 6: 33-40.

Suja JA, del Cerro AL, Page J, Rufas JS, et al. (2000). Meiotic sister chromatid cohesion in holocentric sex chromosomes of three heteropteran species is maintained in absence of axial elements. Chromosoma 109: 35-43.

Ueshima N (1979). Hemiptera II: Heteroptera. In: Animal Cytogenetics. Vol. 3. Insecta, 6. Gebruder Borntraeger, Berlin, $1-117$. 